\title{
Visualization of internal caustics in cylindrical liquid cavities with a pulsed schlieren system
}

\author{
S. MAHAUT ${ }^{(1)}$, D.K. LEWIS $^{(2)}$, G. QUENTIN and M. DE BILLY \\ G.P.S., Universités Paris 6 et Paris 7, Tour 23, 2 Place Jussieu, 75251 Paris cedex 05, France
}

\begin{abstract}
résumé:Des visualisations du champ ultrasonore, produit par réflexion/réfraction d'un train d'ondes planes longitudinales dans une cavité fluide cylindrique à l'intérieur d'un matériau solide élastique, ont été effectuées à l'aide d'un dispositif schlieren en régime pulsé. Les positions des points cuspidés des caustiques formées par réflexions successives dans la cavité sont comparées aux valeurs théoriques calculées géométriquement. Le champ produit par réflexion d'une onde plane sur un demi-miroir cylindrique est également étudié et comparé au champ au voisinage d'une caustique selon l'approximation de Pearcey.

abstract:Visualizations of the acoustic field produced by reflection/refraction of bursts of plane longitudinal waves in the water-filled cylindrical cavity of a solid matrix have been made with a pulsed schlieren system. The positions of the cusp points of the caustics formed by successive internal reflections in the cavity have been compared with their theoretical geometrical values. The acoustic field produced by the reflection of a plane wave on a half-cylindrical mirror has also been studied and compared to the field in the vicinity of a caustic, according to the Pearcey approximation.
\end{abstract}

\section{INTRODUCTION.}

When an acoustic wave is reflected or refracted by a curved interface, cusped caustics are formed. The caustic marks the transition from a region where three rays interfere (inside the caustic) to a region with one ray. Elementary geometrical acoustic theory gives divergent amplitudes in the neighborood of the caustic, thus the wave field has to be evaluated in terms of diffraction integrals. For the simplest case of refraction and reflection in a fluid cylinder, we may calculate the rays paths to determine the shape of the caustic and the locus of the cusp points, whereas we may use the Pearcey function to describe the field in the vicinity of the caustic. We combined both approaches -wave theory and rays theory - to describe our experimental results.

\section{THEORY OF CAUSTICS.}

Consider the one-dimensional diffraction integral of the form [1]

$$
J=\int_{x 1}^{x 2} g(x) \exp [i k f(x)] d x
$$

where the phase function $\mathrm{kf}(\mathrm{x})$ is a real-valued function of the real $\mathrm{x}$, and $\mathrm{k}=2 \pi / \lambda$, where $\lambda$ is the wavelength.If $\mathrm{k}$ is sufficiently large, $\exp [\mathrm{ikf}(\mathrm{x})$ ] oscillates so rapidly compared to $\mathrm{g}(\mathrm{x})$ that cancellation occurs, except in the vicinity of stationary points $\mathrm{x}=\mathrm{xs}$, at which $\mathrm{f}^{\prime}(\mathrm{xs})=0$. As $\mathrm{k} \rightarrow \infty$, only small neighboroods of these points contribute to the integral defined by (1). In practice, there may be several stationary points. The caustic related to two stationary points is the Airy caustic, while three stationary

(1) Permanent address: CEA, Service des Techniques Avancées, CE Saclay, Bâtiment 611, 91191 Gif sur Yvette, France

(2) Permanent address: University of California, Lawrence Livermore National Laboratory, M.E, Adv. Applications, 700 East Avenue, Mail Stop L-495, CA 94551-0808, U.S.A. 
points form a cusped caustic, as first studied by Pearcey [2]. Asymptotic approximations of the integral (1) for each number of stationary points have been summarized in ref [1].

The particular case of three stationary points gives rise to the Pearcey function $\mathrm{P}(\mathrm{X}, \mathrm{Y})$ defined by:

$$
P(X, Y)=\int_{-\infty}^{+\infty}\left[i\left(Y t+X t^{2}+t^{4}\right)\right] d t
$$

where $\mathrm{X}$ and $\mathrm{Y}$ are dimensionless variables describing the shape of the caustic near the cusp, i.e,

$$
Y=\left(\frac{192 \pi^{3}}{\sigma_{\lambda}^{3}}\right)^{\frac{1}{4}} y \quad, X=\left(\frac{12 \pi}{\sigma \lambda}\right)^{\frac{1}{2}} x \quad X^{3}=-\frac{27}{8} Y^{2}
$$

The integral (2) describe the field of a caustic with a cusp point at the origin of the $(x, y)$ axis, where the wave propagates in the $x$ direction, and $\sigma$ is a parameter defining the local shape of the caustic. It is important to notice that the field shows a maximum which is not situated at the origin, i.e at the cusp point, but is displaced at $X \approx 2.2$. FIG4.1 represents the modulus of the Pearcey function. On the left side $(X<0)$, the broken curve is the caustic curve defined by Eq. (3). Inside this region, each point is made by the contribution of three rays. On the right side, the field in all space is only described by one ray.

One can also determine geometrically the shape of the caustic by calculating the rays paths in the simple case of refraction and internal reflections of a plane wave in a cylindrical cavity. As the caustic is defined as the transition between a region of three rays and a region of one ray, one may calculate the locus where two adjacent rays intersect to form the caustic. This method has been used in optics to study the internal reflections in illuminated liquid droplets [3]. We applied these calculations to determine the positions of the cusp points and the local shape of the caustic. A plane wave propagates along the $X$ axis in a solid which longitudinal wave speed is $\mathrm{Cl}$ and intersect a fluid filled cylindrical cavity of radius a situated at the origin of $(x, y)$ axis, of sound speed $\mathrm{C} 0$. If we define the 'p-ray family' as the envelope of rays that have made $\mathrm{p}-1$ internal reflections inside the cavity, the cusp points of the caustics have the coordinates:

$$
X_{c}=\frac{(-1)^{p}}{2 p-1-n} a, Y_{c}=0
$$

Where $\mathrm{n}$ is the index of refraction : $n=\frac{C_{l}}{C_{0}}$

\section{THE EXPERIMENTAL SETUP.}

We used a pulsed schlieren system to visualize the acoustic field.Figure 1 shows a schematic representation of our experimental setup. The schlieren technique is an acousto-optical technique where an expanded He-Ne laser beam is diffracted by an acoustic wave propagating in a fluid. An optical system is used to focus the light rays on a black stop, so that only the diffracted rays, thus the image of the acoustic wave fronts, can hit the camera. We used a Bragg cell (acousto-optical modulator) to have a pulsed laser beam, which enables us to observe the acoustic wave at different transit times, by changing the time delays between the acoustic pulsed emission and the opening of the Bragg cell. More informations about this method may be found in ref [4].

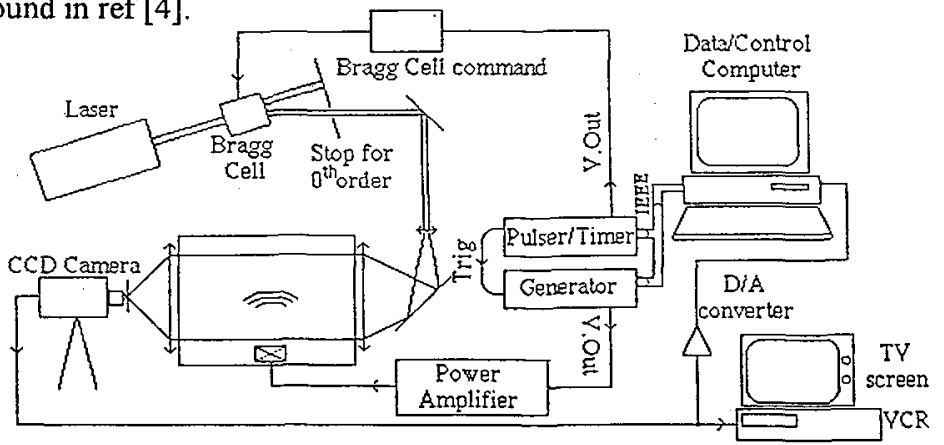

FIG.1. Schematic diagram of the experimental setup 


\section{EXPERIMENTAL RESULTS AND DISCUSSION.}

\subsection{Caustics made by internal reflection in a fluid cavity}

We first studied the internal reflection in fluid-filled cylindrical cavities. We insonified, at normal incidence, different solid matrix (duraluminum, brass and teflon) immersed in a water tank. The cylindrical cavity is placed in the axis of the laser beam, and the acoustic beam propagates along the $X$ axis, perpendicular to the light beam to assure the orthogonal acousto-optical interaction. We used transducers in pulsed mode, and a range of excitation between 5 and $10 \mathrm{MHz}$ to assure the validity of geometrical acoustics, i.e for large $\mathrm{ka}$ (where $\mathrm{k}$ is the wave number and a the radius of the cylindrical cavity).

FIG 2.1 and 2.2 show the schlieren pictures of the first two caustics for a solid matrix of duraluminum ( $n=4.19$ ) with a $5 \mathrm{MHz}$ transducer, whereas FIG 3.1 and 3.2 represent the rays paths associated to this index of refraction (from ref [3])

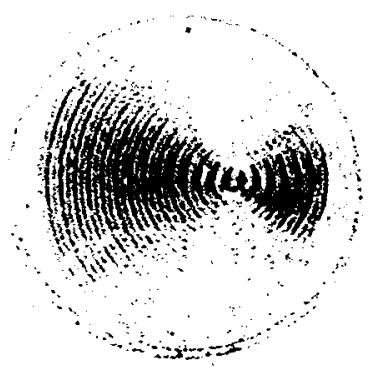

FIG 2.1 First caustic $(p=1)$

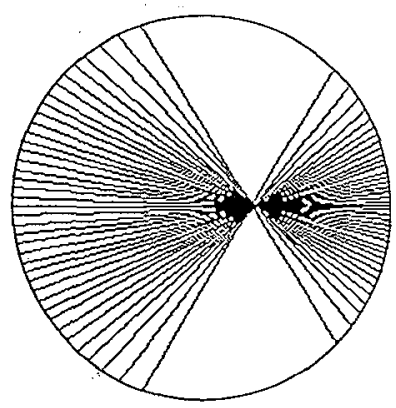

FIG $3.1 p=1$ family rays

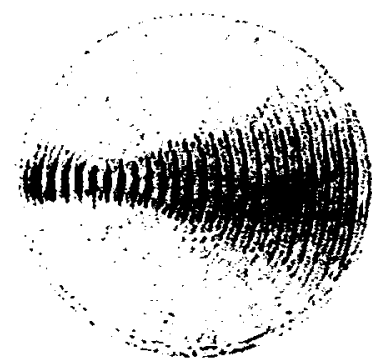

FIG 2.2 Second caustic $(\mathrm{p}=2)$

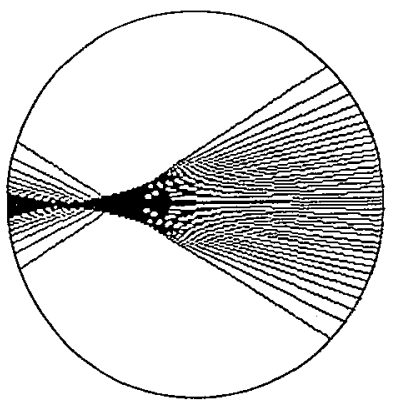

FIG $3.2 \mathrm{p}=2$ family rays

The table below shows the comparison between the theoretical and measured X/a coordinates of the cusp points. It has to be noticed that the measured positions of the cusp points correspond to the inversion of phase of the wave fronts, not to the highest intensity. The agreement is reasonably good, especially if we consider that the second caustic doesn't exhibit a very sharp cusp, so the measurement can't be precise. It can also be pointed out from our experiments that the difference between both values increases with the number of internal reflections, which probably means that the incident wave is not really a plane wave, and thus gives rise to errors in the calculations of the rays paths for high orders.

\begin{tabular}{|c|c|c|}
\hline $\mathrm{p}$ (order of the caustic) & theoretical & Experimental \\
\hline 1 & 0.31 & 0.33 \\
\hline 2 & -0.84 & -0.63 \\
\hline
\end{tabular}




\subsection{Caustic made by reflection on a cylindrical mirror}

We also used a half-cylindrical mirror to observe the reflection of the acoustic wave on a curved interface. The Eq. (4) is no longer valid, but it can be easily shown that the cusp point is situated at $\mathrm{Xc}=\mathrm{a} / 2$, where $\mathrm{a}$ is the radius and the wave is propagating along the positive $\mathrm{X}$ axis.

The FIG 4.1 shows the modulus of the Pearcey function defined by Eq. (1), while FIG 4.2 shows the schlieren image of the reflection of the acoustic wave on the mirror.This picture represents only the reflected wave : the interferences we observe in the vicinity of the cusp, producing local maximas and minimas of intensity, can only be described as the interference between adjacent reflected rays. The agreement between the acoustic field and the Pearcey function seems to be good, assuming we should compute the Pearcey function with the exact geometrical parameter $\sigma$ to fully describe our acoustic field.
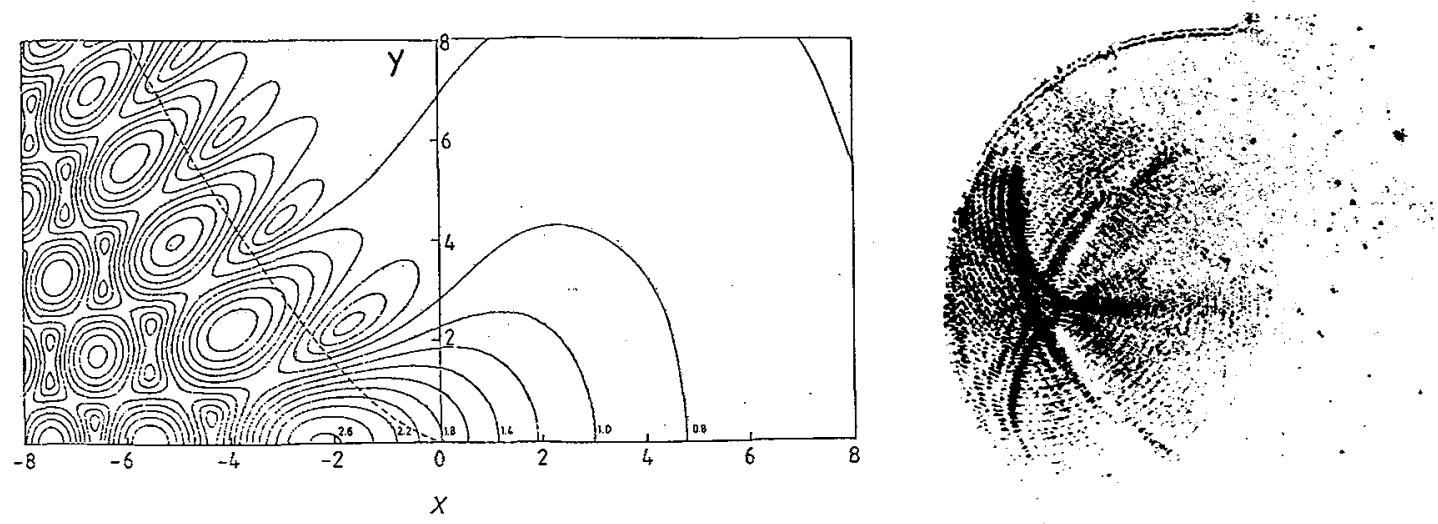

FIG 4.1 The modulus of the Pearcey function ( from ref [1])
FIG 4.2 Visualization of the reflection of the plane wave

\section{Conclusion}

We presented visualizations of cusped caustics and compared it to the rays paths and the acoustic field approximation of Pearcey. It should be noted that the structure of the caustic in the cylindrical cavity doesn't seem to fit the Pearcey approximation, whereas the direct reflection on the mirror shows a close agreement. We suggest that this phenomenon can be explaned in this way : the refraction coefficient of rays for high angles of incidence shows that they cannot contribute sufficiently to the caustic -compared to the rays close to the axis, whereas the reflection coefficient for the mirror shows that the rays of high angles of incidence contribute to a large extent to the caustic. The schlieren system turns out as a very valuable tool to visualize and measure the acoustic field in all space.

\section{References}

[1] Stamnes J. Waves in Focal Regions (Adam Hilger, Bristol 1986) pp 91-135.

[2] T. Pearcey, The structure of an electromagnetic field in the neighborhood of a cusp caustic, Philos. Mag. 37 (1946) 311-317.

[3] Lock J.A. and Hovenac E.A, Internal caustic structure of illuminated liquid droplets,

J. Opt. Soc. Am. A8 (1991) 1541-1552.

[4] N Gespa, La Diffusion Acoustique (Cedocar, Paris 1987) pp 621-638 